\title{
Der gute Staat
}

\author{
The Good State
}

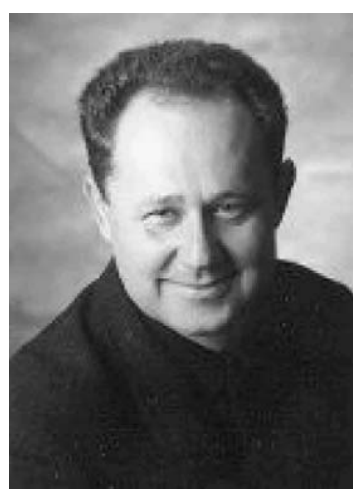

Prof. Dr. Manfred Wildner
Bibliografie

DOI $10.1055 / \mathrm{s}-0029-1220745$

Gesundheitswesen 2009;

71: 263-264

(c) Georg Thieme Verlag KG

Stuttgart · New York

ISSN 0941-3790

Korrespondenzadresse

Prof. Dr. M. Wildner

Bayerisches Landesamt für

Gesundheit und Lebens-

mittelsicherheit

Veterinärstraße 2

85762 Oberschleißheim

manfred.wildner@lgl.bayern.de
„Wenn der Meister regiert, ist sich das Volk kaum bewusst, dass es ihn gibt. Der Zweitbeste ist ein Führer, den man liebt" [1]. Diese Worte sind uns vom Begründer des Taoismus, dem „Alten Meister“ (Übersetzung von „Lao-Tse“, 6. Jhdt. v. Chr.) überliefert. Ob es ihn als Person wirklich gegeben hat? Möglich ist, dass diese Unsicherheit eine Folge seiner gelebten Lehre ist: seine Ämter in Namenlosigkeit auszuüben, um dem Tao nicht im Weg zu stehen. Dem Tao? Wörtlich übersetzt bedeutet es „der Weg“. Gemeint ist ein transzendentes Prinzip höchster Wirklichkeit und Wahrheit, dass aus unserer sichtbaren, in sich zerteilten „Welt der zehntausend Dinge“ heraus rational nicht zu erfassen ist. In der japanischen Wortform „Do“ ist es auch im Westen angekommen. Ju-do, Sa-do, Zen-do - der sanfte Weg (als Kampfkunst), der Weg des Teetrinkens, der Weg des StillSitzens. Der Taoismus hat viel zur erstaunlichen Stabilität des seit Jahrtausenden bestehenden chinesischen Staatsgebildes beigetragen.

Insbesondere der Weg des „Still-Sitzens“ scheint weit entfernt von moderner westlicher politischer Praxis zu sein. Und was die zurückgenommene Rolle des Staats betrifft: Ist nach den finanzpolitischen Katastrophen eines reduzierten neoliberalen Staatsverständnisses nicht schon wieder ein Ruf nach mehr Staat zu vernehmen? Vielleicht sogar, nach dem empfundenen Versagen der „unsichtbaren Hand“ klassischer marktwirtschaftlicher Theorie [2], ein Ruf nach einer spürbaren „starken Hand“ des Staats? Ein Heilsversprechen, dem sich die politisch bewussten Schichten in der Bundesrepublik mit ihrem noch wachen historischen Bewusstsein verweigern dürften.

Auch innerhalb der Ärzteschaft sind im Zuge der Honorarverteilungskämpfe Parteiungen und Risse entstanden. Wie lässt sich der bedrohte und teilweise verlorene soziale Frieden wieder erlangen? Welche Rolle kommt dabei dem Staat zu? Was ist überhaupt „Staat“, was sind seine Aufgaben und wie soll er sie wahrnehmen? Dass auf diese Fragen vielfältige Antworten möglich sind und auch in der Geschichte und in den Kulturräumen bis heute gegeben werden, ist schon die erste Antwort [3]. Landesverteidigung? Nicht zwingend eine Staatsaufgabe, wie uns private Militärunternehmer von Wallenstein bis Executive Outcomes lehren. Finanzverwaltung? Jakob Fugger (1459-1525) würde über eine vermeint- liche Unabhängigkeit des Staates von der Wirtschaft nur lächeln [4]. Strafvollzug? In den USA mit ihrer hohen Quote an Gefängnisinsassen bei den Männern fast $1 \%$ der Bevölkerung - sind über 100000 in privaten Gefängnissen untergebracht, in Deutschland hat der private Strafvollzug ebenfalls Einzug gehalten [5]. Den Staat mit seinen formalen Institutionen und Gesetzen gleichzusetzen, ist seit den Verbrechen der nationalsozialistischen Unrechtsherrschaft nicht mehr vertretbar. Augustinus (354-430) beklagt die Entartung eines solchen Staatsverständnisses schon im 5. Jahrhundert: „Was anders sind also Reiche, wenn ihnen Gerechtigkeit fehlt, als große Räuberbanden?" [6].

Die Klage des Augustinus führt uns zur Frage, wie der Staat - und auch die Ordnung der kleineren sozialen Lebenswelten - beschaffen sein soll. Justitia et pax, Gerechtigkeit und Frieden, waren lange Zeit die Essenz staatlicher Daseinsberechtigung. Dass diese beiden Qualitäten zusammenhängen, ist altes Wissen. „Gerechtigkeit schafft Frieden", mit diesem biblischen Jesaja-Wort $(32,17)$ beschwor Papst Pius XII. die Diktaturen vor Ausbruch des 2. Weltkrieges vergeblich. „Der Abt [...] treffe keine ungerechte Verfügung, als könnte er seine Macht willkürlich gebrauchen", schreibt auch der Ordensgründer Benedikt von Nursia im 6. Jahrhundert [7]. Wie lässt sich staatliche Macht in einem säkularen Verständnis gegenüber seinen Bürgern begründen? Staatliches Gewaltmonopol im Dienst des sozialen Friedens ist ein prominenter legitimatorischer Grundgedanke verschiedener Vertragstheorien nicht erst seit Thomas Hobbes' „Common-Wealth“ [8]. Zur Entwicklung des Staatsgedankens sei auf die reichliche diesbezügliche Literatur von berufener Seite verwiesen. Heute umfasst die für Frieden zentrale Gerechtigkeitsdiskussion viele Facetten, u.a. wirtschaftliche Gerechtigkeit, soziale Gerechtigkeit, Chancengerechtigkeit hinsichtlich Bildung, Einkommen, Berufschancen und Gesundheit.

Für die Moderne grenzt der amerikanische Philosoph John Rawls (1921-2002) Gerechtigkeit als „Fairness“ von nur utilitaristischen Nützlichkeitskalkülen ab. Sein Konzept stützt sich auf Freiheit, Gleichheit und Belohnung für Dienste am Allgemeinwohl [9]. Prinzipien, die an Aktualität nichts zu wünschen übrig lassen. Ein Modell dazu ist unser heutiger liberaler, auf Freiheitsrechten ge- 
gründete Staat. Schwachstelle dieses säkularisierten freiheitlichen Staatsmodells ist die Loslösung von seinen vorpolitischen Grundlagen, welche traditionell mit religiös geprägten bzw. religiös stabilisierten Werten einer Gesellschaft in Verbindung gebracht werden. Diese Problematik hat der deutsche Verfassungsrichter Ernst-Wolfgang Böckenförde $\left({ }^{*} 1930\right)$ in dem nach ihm benannten Diktum auf den Punkt gebracht:,Der freiheitliche, säkularisierte Staat lebt von Voraussetzungen, die er selbst nicht garantieren kann. Das ist das große Wagnis, das er, um der Freiheit willen, eingegangen ist. Als freiheitlicher Staat kann er [...] nur bestehen, wenn sich die Freiheit, die er seinen Bürgern gewährt, von innen her, aus der moralischen Substanz des einzelnen und der Homogenität der Gesellschaft, reguliert.“ [10].

Ist dieses Wagnis einer sich „,von innen her“ regulierenden bürgerlichen Freiheit naiv? Ist nicht die Wechselbeziehung zwischen „einem listigem Staat, der durch ein intransparentes Steuersystem, Scheinversicherungen und Paragraphendschungel den Zusammenhang zwischen seinen Einnahmen und Ausgaben undurchschaubar macht, und dem schlaumeierischen Bürger, der sich durch Ausnützen aller formal erschließbaren Anspruchsquellen an seinen Mitbürgern schadlos zu halten versucht", Realität geworden [3, S. 39-40]? Sind Homogenität und moralische Substanz nicht längst im postmodernen Werte-Sammelsurium verloren gegangen? Vielleicht doch nicht - nach der Postmoderne scheint sich leise ein Postmaterialismus zu etablieren, im Zuge eines von Ronald Inglehart $\left({ }^{*} 1934\right)$ postulierten Wertewandels nach erfolgreicher Befriedigung der Grundbedürfnisse [11]. Inhalte eines solchen Wertewandels? Statt erhöhtem Konsum eine bewusste Orientierung hin zu „postmateriellen” Werten wie politischen Freiheiten, Umweltschutz, Sinnsuche - auch Gesundheit. Ihre Vertreter werden transatlantisch als Lohas (lifestyle of health and sustainability), Lovos (lifestyle of voluntary simplicity) oder Scuppies (socially conscious upwardly-mobile people) bezeichnet.

Wie der Soziologe Niklas Luhmann (1927-1998) darlegt, sind moderne Gesellschaften von intensiven Dialogen und FeedbackSchleifen geprägte, selbstreferentielle Systeme [12]. Diese Sicht hat sehr gute Übereinstimmung mit sich entwickelnden alternativen gesellschaftlichen Lenkungsformen, welche mit „(new) governance“ bezeichnet werden: Netzwerke, Partnerschaften, Pakte, indirekte Einflussnahmen, komplexe Wechselspiele zwischen Regierungen und Nicht-Regierungsorganisationen - also multiple Akteure, Verantwortlichkeiten und Beziehungen in einer komplexen Konstellation von Staat, Medien und Wirtschaft. Mut zu einem „konstruktiven Macht-Vakuum, das der streitenden Auseinandersetzung wahrheitstiftenden und traditionsbegründenden Spielraum gewährt": So sieht der Philosoph Walter Schweidler die „Kontinuität europäischer politischer Ethik von den griechischen Anfängen bis heute " $[3$, S. 22]. In dieser Tradition stand vor ihm auch Winston Churchill mit seinem Ausspruch im britischen Unterhaus vom 11. November 1947: „Democracy is the worst form of government, except for all those other forms that have been tried from time to time" [Dt.: Demokratie ist die schlechteste Regierungsform - außer all den anderen Formen, die von Zeit zu Zeit ausprobiert worden sind].
Governance-Ansätzen im Feld von Gesundheit? „Shared Decision Making“ und mündige Patienten, „Nouvelle Gouvernement“ bei der Krankenhausplanung, öffentliche Diskurse zu Impfungen, zur Zeugung und zum Ende des Lebens, Kollegialsysteme statt starrer (chef-)ärztlicher Hierarchien, indirekte Steuerungen durch überwachte Eigenkontrolle statt direkte Fremdkontrollen, HACCP-Systeme u.v.a.m. Künftig werden vermehrt Systemwissen und Systemintelligenz gefragt werden - die große Herausforderung an die Public Health-nahen Wissenschaften. Governance: Eine moderne Vorstellung? Vielleicht - und in gewissem Sinn gleichzeitig sehr nahe an der Vorstellung des „Alten Meisters“ Lao-Tse: „Wenn sein [des Meisters] Werk getan ist, sagt das Volk: ,Unglaublich: Wir haben es ganz allein vollbracht“" [1].

Uns westlichen Menschen wird zunächst nichts anderes übrig bleiben, als uns in der „Welt der zehntausend Dinge“ weiter wacker zu bemühen. Als Hilfe dafür sind die Beiträge dieses Heftes gedacht: Sie beziehen sich auf Hygieneregeln im Krankenhaus und Schadstoffbelastungen in unseren weitgehend selbstgemachten Lebens(um)welten, auf die Bewältigung der AlzheimerDemenz als einer der großen Herausforderungen in Begleitung einer steigenden Lebenserwartung, auf Krebsregistrierung, auf das DRG-Krankenhausfinanzierungssystem und auf epidemiologische Methoden für den bevölkerungsbezogenen Gesundheitsschutz. Auch diese, den Lesern wohl vertrauten, Wege führen bisweilen zu „Meisterschaften“ - mit Lernbereitschaft, Geduld und Ausdauer.

\section{Literatur}

1 Laotse. Tao Te King. Eine zeitgemäße Version für westliche Leser. Kapitel 17. Goldmann, München, 2003; S. 29

2 Smith A. An Inquiry into the Nature and Causes of the Wealth of Nations, Band 1 (1776) Nachdruck von 1981, Indianapolis, Indiana, USA, S. 14f. [Deutsch: Recktenwald HC. Adam Smith. Der Wohlstand der Nationen: Eine Untersuchung seiner Natur und seiner Ursachen. Deutscher Taschenbuch Verlag, München, 2003]

3 Schweidler W. Der gute Staat. Reclam, Stuttgart; 2004

4 Ogger G. Kauf dir einen Kaiser. Die Geschichte der Fugger. Knaur, München, 1979

5 Koch M. Private Gefängnisse profitieren von der Krise URL: http://www. sueddeutsche.de/wirtschaft/617/453309/text/ [Zugriff am 08.03.2009]

6 Augustinus A. Der Gottesstaat (De civitate dei). Liber IV, 4. Johannes Verlag, Einsiedeln, 1961; S. 115

7 Salzburger Äbtekonferenz, Hrsg. Die Benediktusregel, Kap. 63. Beuroner Kunstverlag, Beuron, 1992; S. 219

8 Hobbes T. Leviathan, The Matter, Forme and Power of a Common Wealth Ecclesiasticall and Civil (1651) URL: http://www.gutenberg. org/etext/3207 [Zugriff am 14.03.2009])

9 Rawls J. Justice as fairness. Philosophical Review 1958; 67 URL: http:// www.hist-analytic.org/Rawlsfair.htm [Zugriff am 14.03.2009]

10 Böckenförde EW. Staat, Gesellschaft, Freiheit. Frankfurt, 1976; S. 60

11 Inglehart $R$. The silent revolution in Europe. Intergenerational change in post-industrial societies. The American Political Science Review 1971; 65 (4): 991-1017

12 Luhmann N. Soziale Systeme: Grundriss einer allgemeinen Theorie. Suhrkamp, Frankfurt/M., 1984, 2008 